\title{
Two memories for geographical slant: Separation and interdependence of action and awareness
}

\author{
SARAH H. CREEM and DENNIS R. PROFFTTT \\ University of Virginia, Charlottesville, Virginia
}

\begin{abstract}
The present study extended previous findings of geographical slant perception, in which verbal judgments of the incline of hills were greatly overestimated but motoric (haptic) adjustments were much more accurate. In judging slant from memory following a brief or extended time delay, subjects' verbal judgments were greater than those given when viewing hills. Motoric estimates differed depending on the length of the delay and place of response. With a short delay, motoric adjustments made in the proximity of the hill did not differ from those evoked during perception. When given a longer delay or when taken away from the hill, subjects' motoric responses increased along with the increase in verbal reports. These results suggest two different memorial influences on action. With a short delay at the hill, memory for visual guidance is separate from the explicit memory informing the conscious response. With short or long delays away from the hill, short-term visual guidance memory no longer persists, and both motor and verbal responses are driven by an explicit representation. These results support recent research involving visual guidance from memory, where actions become influenced by conscious awareness, and provide evidence for communication between the "what" and "how" visual processing systems.
\end{abstract}

Hills appear steeper than they actually are, an intriguing phenomenon recently described by Proffitt, Bhalla, Gossweiler, and Midgett (1995). For example, a $5^{\circ}$ hill is consistently judged to be about $20^{\circ}$. Although people judge hills to be much steeper than they actually are, they can traverse them with no difficulty. Despite an exaggeration in conscious perceptual judgment, people do not manifest this overestimation in a visually guided action directed toward the hill. Proffitt et al. showed that a motoric (haptic) adjustment of slant did not reflect the overestimation seen in conscious reports of the hill inclination. They proposed that the perceived exaggeration of geographical slant preserves the relationship between distal inclination and people's behavioral potential. The steepness of a hill that one perceives gives the observer information about the effort required to traverse that hill. Furthermore, Proffitt et al. suggested that the differences between verbal and motoric estimates of hill slants illustrate the separation of the visual pathways that mediate conscious perception and visually guided action.

We recently asked people to judge the slants of familiar hills from memory. We found that people remembered hills to be even steeper than they had perceived them to be.

This research was supported by NIMH Grant MH52640 and NASA Grant NCC-2-925 to D. R. P. The authors wish to thank Mukul Bhalla for many discussions of ideas, Arthur Shulman for providing valuable comments on an earlier version of this paper, and Valerie Lewis and Gavin Sacks for their help in running the experiments. Correspondence should be addressed to S. H. Creem, Department of Psychology, 102 Gilmer Hall, University of Virginia, Charlottesville, VA 22903-2477 (e-mail: creem@virginia.edu).
A question followed this finding: Given this increase in conscious overestimation, what will happen to the motoric estimate? The present experiments yielded two principal findings. First, people showed a consistent increase in conscious overestimation of hill slants in memory over several different time delays. Second, memorial motoric responses differed depending on the length of delay between perception and response and the context in which the response was made. With a short delay, motoric responses made in the context of the hill remained the same as those made when perceiving the hill. Away from the surroundings of the hill, both short and long delays led to an increase in motoric responses along with verbal overestimations.

Our findings show that explicit memory for geographical slant, as expressed through a verbal response, increased in comparison with the perception of hill slant. For motor responses, two memory systems are implicated: a visual guidance memory effective for short delays within the context of the hill, and an explicit memory effective for long delays and short delays away from the setting of the hill. Given a short delay within the presence of the hill, an implicit memory for action and an explicit memory remain dissociated in informing motoric and verbal responses, respectively. Memory for action guides the motoric response, whereas explicit memory informs the verbal response. With longer delays, as well as short delays away from the hill environment, both motoric and verbal responses are informed by explicit memory, suggesting communication between the two visual systems. The results for long and short delays away from the hill are consistent with the recent literature that visually guided actions from memory are informed by an explicit stored representation (Rossetti, in press). 


\section{Geographical Slant Perception}

Geographical slant is defined as an inclination relative to the surface of the earth (Gibson, 1979; Gibson \& Cornsweet, 1952). In contrast, optical slant is specified relative to the frontal plane perpendicular to the line of sight (Gibson, 1979). Until recently, only a few studies had investigated geographical slant perception, and these showed there to be a tendency for people to perceive the steepness of terrain to be greater than it actually is (Kammann, 1967; Ross, 1974). Although the incline of hills is consciously overestimated, Kinsella-Shaw, Shaw, and Turvey (1992) found that people accurately perceive the affordance of hills. Subjects accurately judged the maximum slant they could ascend and adjusted a viewed platform to match the inclination of a ramp felt by their foot.

An extensive study on geographical slant perception was completed by Proffitt et al. (1995) in which subjects provided three response measures: verbal, visual, and haptic. The verbal measure was a verbal report of the subject's perception of the incline of the hill. The visual measure was a matching task, in which the subject was instructed to adjust a disk that represented the cross section of the hill. The disk consisted of an adjustable angle that could be set to the perceived inclination of the hill. The haptic measure was given on a tilt board, adjusted by the subject's palm without looking at the hand, until the subject felt that the incline was equal to that of the hill. Standing at either the top or the bottom of the hill, subjects overestimated the incline of the hill when giving a verbal or visual report, but they were much more accurate when giving the haptic estimate. Slant judgments were found to conform to power functions having exponents less than 1 , thereby showing compression. The functional virtue of magnitude estimation and concomitant response compression is that it affords a heightened sensitivity to small changes in slant for small slants. The human potential to traverse hills is limited to small angles. Proffitt et al. related the differences between verbal/visual and haptic responses to the different purposes of conscious awareness and motoric actions. The perceived exaggeration in conscious slant perception provides information to help inform us about the possibility of traversing a given hill. However, the act of traversing an incline demands a veridical evaluation of the environment. Long-term planning is a task of the conscious perception system, whereas immediate visually guided behavior is not. The accuracy of the haptic measure suggests that visually guided actions are immune to slant overestimations. Although people may perceive a hill to be much steeper than it is, their feet smoothly walk over the slanted terrain, without any awareness of contradictions in their responses.

Proffitt et al. (1995) further showed that behavioral potential influenced conscious overestimation. They asked subjects to view hills while standing at the top. A $30^{\circ}$ hill may be ascended, whereas it is impossible to walk down from the top. Accordingly, steeper hills appeared steeper from the top than from the bottom. A second manipulation of behavioral potential was fatigue. Subjects judged hills before and after an exhausting run. Slant overestimation became even more exaggerated after the run, when the person was fatigued. However, the motoric adjustment was unaffected. Furthermore, Bhalla and Proffitt (1997) found that conscious overestimation increased with a number of additional behavioral potential manipulations, such as wearing a backpack or being of low physical fitness, elderly, or in declining health. All of these manipulations influenced perceived steepness of hills but not the visually guided action measure.

A second part of these studies involved a measure of internal consistency, showing that, although they might appear to be separated, phenomenal awareness and visually guided action do communicate. Subjects were asked to make haptic estimates on the tilt board in response to verbal instructions. The haptic adjustment made in response to a given verbal instruction was internally consistent with responses given while hills were being viewed. For example, when subjects viewed a $10^{\circ}$ hill, they called it $30^{\circ}$ and adjusted the board to about $10^{\circ}$. When the subjects were not looking at the hill and were simply told to set the board to $30^{\circ}$, they set the board to $10^{\circ}$ again. Thus, an internal consistency was found between verbal representations and the haptic adjustments.

\section{Two Visual Systems of Processing}

Proffitt et al. (1995) interpreted the dissociation between visual awareness and motoric action found in geographical slant to be consistent with the neuroanatomical and functional segregation of the visual system. Two visual pathways project from the primary visual cortex: a ventral stream leading to the inferotemporal cortex, and a dorsal stream leading to the posterior parietal cortex. Traditionally, this structural distinction in visual processing has been labeled functionally as "what" and "where" (Ungerleider \& Mishkin, 1982). It has been thought that the dorsal stream processes spatial location, whereas the ventral stream processes object features. More recently, these pathways have been proposed to support "what" and "how" functions, in which the ventral stream subserves conscious visual awareness and the dorsal stream subserves visually guided actions (Goodale \& Milner, 1992; Milner \& Goodale, 1995). This account proposes that both streams process similar information about object features and spatial relationships. The difference between the two pathways lies in their transformations of the information. This explanation focuses on differences in output rather than the incoming information. Accordingly, the ventral stream uses visual information to process object features and long-term representation, whereas the dorsal stream acts to transform the information immediately in preparation for action.

Studies of the "what" and "how" visual processing systems in humans have focused on both immediate responses and delayed responses to visual stimuli. Several behavioral studies involving immediate responses have shown evidence for the segregation of visual awareness and visually guided action. Bridgeman and his colleagues (Bridgeman, Kirch. \& Sperling, 1981; Bridgeman, Lewis, 
Heit, \& Nagle, 1979) asked subjects to judge verbally and through pointing or saccadic eye movements whether a target was displaced. In several experiments, subjects were able to make saccades and point accurately to a displaced target, when they did not verbally report that they detected the displacement. These results suggest a dissociation of what the authors label cognitive and motor systems. Pelisson, Prablanc, Goodale, and Jeannerod (1986) found similar results in another pointing task. Subjects were able to adjust their pointing finger to a change in target location without conscious awareness of the displacement of the target. They tracked the movement accurately without being aware that there was any movement.

A more recent study has shown this dissociation using visual illusions (Aglioti, DeSouza, \& Goodale, 1995). Subjects were presented with the Ebbinghaus illusion, in which two same-sized disks appear to be different sizes depending on the size of the surrounding circles. In this study, subjects made the perceptual error of deciding that the target disk surrounded by smaller disks was larger than the target disk surrounded by larger ones. However, when the subjects were asked to pick up the disks, they scaled their grip to the actual size of the target disk rather than the perceived size. It also should be noted that the accuracy of the subjects was not a result of visual error correction in which they were simply comparing their grip aperture with the diameter of the disk while performing the movement. Aglioti et al. (1995) stated that, in this study and numerous others, it has been shown that the calibration of maximum grip aperture is mostly determined by a motor program before the action even takes place. The maximum grip aperture, although larger than the diameter of the disk, correlated well with the size of the target. Here again is a dissociation between what is consciously perceived and the action performed toward the object.

Further evidence for separate "what" and "how" streams comes from neurological patients. One patient, D.F., who has visual form agnosia has been studied extensively (Goodale, Jakobson, et al., 1994; Goodale, Milner, Jakobson, \& Carey, 1991). Damage in the ventrolateral region of the occipital cortex as a result of carbon monoxide poisoning has led to her inability to discriminate or recognize simple objects. Several studies with D.F. have shown that she has accurate egocentric visuomotor abilities, despite her extreme deficits in object recognition. When presented with different-sized blocks, her performance in reaching for and grasping the objects matched normal subjects' reaching behavior. The size of her anticipatory grasp was highly correlated with the width of the block. However, when asked to indicate with her finger and thumb how wide the block was, her responses were unrelated to the block dimensions. Similarly, she could not discriminate the orientation of a slot, but she could accurately "post" a solid plaque into the slot when involved in a motor response (Goodale et al., 1991). These studies suggest that-although D.F. is not able to perceive object characteristics or location-when asked to act upon an object, she accommodates to these dimensions accurately.
Evidence from agnosics such as D.F. and from patients with optic ataxia (Jakobson, Archibald, Carey, \& Goodale, 1991), who as a result of damage to the posterior parietal cortex fail to reach and grasp accurately but can identify objects well, has led to a clear double dissociation. Those with temporal damage cannot recognize or consciously "perceive" objects, but they can accurately act on them; those with parietal damage cannot accurately perform visually guided actions, although they have little problem with explicit object recognition.

Although immediate responses to a visual stimulus show a dissociation between phenomenal awareness and visually guided action, we might predict a different result for delayed responses when on-line visual input is no longer available. Rossetti (in press) stressed the importance of time factors in the dissociation between the two visual processing streams. He discussed several examples of normal subjects and brain-damaged patients that support the claim that the system informing visually guided action is constrained by time. In general, Rossetti showed that motoric performance degrades rapidly with time and that, when delays are added to a visually guided action task, actions require information from awareness. For example, blindsight patients can, with encouragement, direct an action to a stimulus in their blind hemifield without "seeing" the stimulus. Rossetti found that a blindsight patient, N.S., was better at performing an action task when her movement latency decreased, suggesting that information needed for action is only available for a short time. Other blindsight patients have also shown that a quicker movement led to more accurate action (Rossetti, in press).

A similar finding has been shown in a reaching task from memory with the agnosic patient, D.F. (Goodale, Jakobson, \& Keillor, 1994). As already mentioned, although D.F. cannot recognize objects, she reaches and grasps for them accurately. Goodale, Jakobson, and Keillor (1994) explored the nature of stored representations by testing normal subjects and D.F. on pantomimed reaching actions. Normal subjects viewed an object, and they were asked to reach for it $2 \mathrm{sec}$ later while wearing opaque glasses that prevented sight of the object. They found differences when comparing the kinematics of reaching from memory to the kinematics of reaching with vision. In a second condition, the object came back into sight at the end of the 2sec period, and the subjects reached as they had with normal visually guided action. This result suggests that visuomotor coordinates are computed immediately before each action.

To further understand the system driving these pantomimed actions, a second set of experiments was conducted with agnosic patient D. F. Goodale, Jakobson, and Keillor (1994) proposed that if the action system works only in real time, with continuous updating, then the stored information driving the action from memory must depend on the system designed for representing objects over longer periods of time. This system, they propose, is the conscious perception system driven by the ventral pathway to the inferotemporal lobe. Assessing D.F., who has 
severe temporal damage and little or no ability to consciously perceive objects, on her ability to pantomime actions from memory would provide evidence for this proposal. In fact, the study showed that D.F.'s reaching from memory was completely different from her normal reaching. The normal subjects showed kinematic differences, but some accuracy, in grasping from memory, whereas D.F.'s grasp did not accommodate to the target in any way. These findings are strikingly different from D.F.'s performance with the stimulus present. She, like normal subjects, shapes her hand to the size and dimensions of the target object as it approaches the object. However, in memory, all anticipatory hand shaping disappeared for D.F. With consideration, this finding is not surprising because D.F. did not have the object in front of her to drive the visuomotor system acting in real time. Unlike normal subjects, she also could not refer back to stored representation of the object because she had no "percept" of the object from the beginning. Thus, D.F. had no representation to drive her action, as a normal subject would do from memory.

Several other studies in particular have examined the distinction between visual awareness and visually guided action with tasks involving a delay between perception and response. Wong and Mack (1981) compared saccades made to a displaced target immediately with saccades to a remembered visual position. As in the Bridgeman experiments, immediately after the presentation of the target, saccades were accurately directed to the actual displacement of the target even though the perceived displacement differed. However, when subjects were then asked to look back to the original location of the target, the saccades were made to the perceived location rather than the actual location. These memory-driven saccades were based on the subject's perception of the displacement, in sharp contrast to the immediate saccades, which were driven by retinocentric coordinates. These findings suggest that in memory, a stored perceptual representation must be accessed that then informs the action from an environmental rather than egocentric frame of reference.

More recently, Bridgeman, Peery, and Anand (1997) reached conclusions very similar to those of Wong and Mack (1981). They created a situation based on the Roelofs effect, in which people tend to misperceive the position of a target in an unstructured field. They presented subjects with a rectangular frame centered either on their midline or $5^{\circ}$ left or right of center, with an " $x$ " placed inside the frame in one of five positions. Without a delay, they found that, with a "cognitive" response, subjects misperceived the location of the target in the direction opposite the offset of the frame; however, with a motor (pointing) response, half of the subjects did not show the effect. With a 4-sec delay, all subjects were biased by the frame position in the motoric task. These findings illustrate a motor memory that is short lived, as well as the influence of conscious perception on motor responses after a delay.

Rossetti and his colleagues conducted a series of studies involving pointing to memorized targets. A first experiment tested pointing movements of normal subjects to tar- gets on a computer screen (Rossetti, Lacquanti, Carrozzo, \& Borghese, 1994, cited in Rosetti, in press). They presented two different displays of targets. In each, a target was embedded within a different configuration. An analysis of pointing distributions showed that immediately, distributions of responses were accurate, whereas, with an 8-sec delay, subjects made pointing movements based on the context of the target array. In other words, pointing errors followed the pattern of the array only in the delay conditions. Subsequently, Rossetti and Regnier (1995) conducted a similar task using the proprioceptive modality. With similar targets, a subject's left finger was guided to the target, and a memorial pointing task followed. Similar results were obtained where pointing was influenced by target configuration only in the delay condition. They concluded that pointing from memory becomes dependent on context, whereas immediate movements do not. They suggest that two different frames of reference may be used by the "what" and "how" representations.

These studies of visuomotor behavior to remembered objects not only provide evidence for the division of labor between the "what" and "how" systems but also for the necessary communication of the two streams. For both normal subjects and patients, after a short delay, the action stream appears to falter on its own and needs to refer to the long-term representations in explicit awareness. In normal subjects, this transfer of information is simple, although the arm and eye movements are somewhat altered to follow the explicit perceptual representation. However, for blindsight and agnosic patients who lack the use of their conscious visual processing stream, visually guided action from memory becomes an impossible task.

The "how" system and the "what" system subserve different goals. In order to act in the environment, the action system must constantly update information according to how the environment changes with respect to the observer. This updating requires an egocentric frame of reference sensitive to minute changes in features associated with the target, such as viewer-relative location, orientation, and movement. Because egocentric coordinates of a target can change rapidly, it is necessary to compute the coordinates for action on a momentary basis. This on-line computation requires the presence of the stimulus in the location in which it is perceived. The "what" system serves a different purpose. It acts to encode long-term characteristics of objects. Conscious perception uses a predominantly objector environment-relative reference system where attributes and meanings of objects are encoded in relation to the environment and do not involve momentary egocentric changes needed for action. However, with remembered visually guided actions, there is not a clear division between the two systems. Without on-line information, communication from an explicit representation is needed to inform a motor response. Both systems play a role in executing the action.

Thus, it is seen that the two visual systems can be distinguished by the different reference frames that they employ in carrying out their respective functions. The "what" stream uses both object- and environment-relative reference 
frames, whereas the "how" stream uses egocentric ones exclusively (Milner \& Goodale, 1995; Wraga, Creem, \& Proffitt, in press). This being the case, the particular visual system tapped by each of the response measures used in the present study and in Proffitt et al.'s (1995) experiments can be distinguished by the different reference frames that they require. The verbal and visual responses call for the subjects to judge the slant of the hill with respect to the environmental horizontal, and, thus, these tasks required an environmental reference frame supplied by the "what" system. In contrast, the adjustment made on the tilt board is purely egocentric. Knowledge of the horizontal is completely unnecessary to perform this task. Instead, subjects need only move the hand so as to make it parallel to the slant of the hill. Since the haptic response does not require subjects to relate the incline of the hills to the horizontal, the response can be guided by underlying processes that need not represent geographical slant at all. The haptic response is, thus, represented in an egocentric framework provided by the "how" system.

\section{Overview of the Experiments}

The present series of experiments investigated the effects of time delays and place of response on judgments of geographical slant. Preliminary data suggested that hills seemed steeper to the observer when remembered than when perceived. In the preliminary experiment, subjects responded on a questionnaire, judging the incline of familiar hills. They consistently responded with higher verbal reports than the verbal responses previously collected when subjects were viewing the same hills. In the present study, four experiments were conducted to confirm and elaborate on these preliminary findings. Given that visually guided action is an on-line egocentric process dependent on the presence of the stimulus, we varied both the delay of response and the context in which the response was made. In all of the experiments, subjects were asked to judge three hills from memory, using two measures employed in the previous perception of slant studies: verbal reports and a motoric measure involving haptic adjustments of a tilting board with the unseen hand. Experiment 1 included a short delay between perception and response, within the hill surroundings. Experiments 2 and 3 involved longer delays and responses made necessarily away from the hill. Experiment 4 implemented short time delays as in Experiment 1 but removed the observer from the hill environment.

\section{EXPERIMENT 1 Judgments Made at the Hill After a Short Delay}

Experiment 1 tested whether judgments of slant from memory after a short delay would increase beyond those given when viewing the hills. In this experiment, subjects gave judgments of slant immediately after viewing the hills in two conditions: (1) facing the hill with eyes closed, and (2) turned around. While looking at the hill, subjects were not aware that they would be asked for a slant judgment. Without looking at the hill, subjects gave verbal and haptic estimates of the incline of the hill.

\section{Method}

Subjects. Seventy-five students ( 44 males, 31 females) at the University of Virginia participated. There were 38 subjects in the facing condition, and there were 37 subjects in the turned condition. They were stopped by the experimenter as they walked by the area and asked if they would like to participate. All subjects were naive to the purposes of this experiment and had not participated in any prior slant experiments. None had any apparent locomotor problems.

Stimuli. Three hills on the grounds of the University of Virginia were used as stimuli in the study. These hills were selected on the basis of several criteria. First, they were used in previous slant experiments (Proffitt et al., 1995) so as to provide normative data for comparison. Second, they were near heavy foot traffic areas with familiar and distinct landmarks to allow for easily recognizable descriptions. Third, the hills represented a range of inclinations of $4^{\circ}$, $21^{\circ}$, and $31^{\circ}$, as measured by a Suunto clinometer having an accuracy of $0.5^{\circ}$. The $4^{\circ}$ incline was a sidewalk; the $21^{\circ}$ and $31^{\circ}$ inclines were grassy slopes.

Apparatus. The subjects reported their haptic estimations using a tilt board with a flat palm rest that could be adjusted to match the inclination of the incline. The tilt board was mounted on a tripod whose height was adjusted to slightly above waist level for each subject. The subjects were asked to match the tilt board to the slant of the hill that they were imagining. The tilt board was placed by their side, and they always adjusted it with their dominant hand. The subjects were not permitted to look at their hand while making the adjustments.

Design. Each subject viewed only one hill. In the facing condition, there were 13 subjects for the $4^{\circ}$ hill, 12 for the $21^{\circ}$ hill, and 13 for the $31^{\circ}$ hill. In the turned condition, there were 14 subjects for the $4^{\circ}$ hill, 12 for the $21^{\circ}$ hill, and 11 for the $31^{\circ}$ hill.

Procedure. The subjects viewed the hills binocularly from the front while standing at the base of the hills. They were instructed to look straight ahead and to try to remember the scene that they saw. They were told to be aware of the objects and landmarks in the environment and the layout of the land. The subjects were not explicitly told that they were to remember the slant of the hill, in order to prevent them from attaching an estimate to the incline while they were still looking at it.

After they had looked at the hill for about $10 \mathrm{sec}$, the subjects performed in one of two conditions. In the facing condition, they were told to close their eyes. In the turned condition, they were instructed to turn around $180^{\circ}$ and close their eyes. The tilt board was turned around to face in the same direction as the subject. The subjects were told that they would be asked several questions about what they had just seen. They were asked two questions about the objects in the environment (e.g., "How many light posts did you see in front of you?"). Following these questions, the subjects were asked to imagine the hill that they had viewed and to judge the angle with respect to the horizontal, giving a verbal and a haptic estimate. The verbal report was a numeric estimate of the slant of the hill. The haptic adjustment was made on the tilt board in the manner previously described. The order of verbal and haptic reports was counterbalanced between subjects. The experimenter recorded their responses. Feedback was not given.

\section{Results}

The data from Experiment 1 were compared with the normative data from Proffitt et al. (1995) for the three hills involved in the present set of experiments: $4^{\circ}, 21^{\circ}$, and $31^{\circ}$. The portion of the normative-data set used in these analyses consisted of judgments from 30 subjects for each hill, 


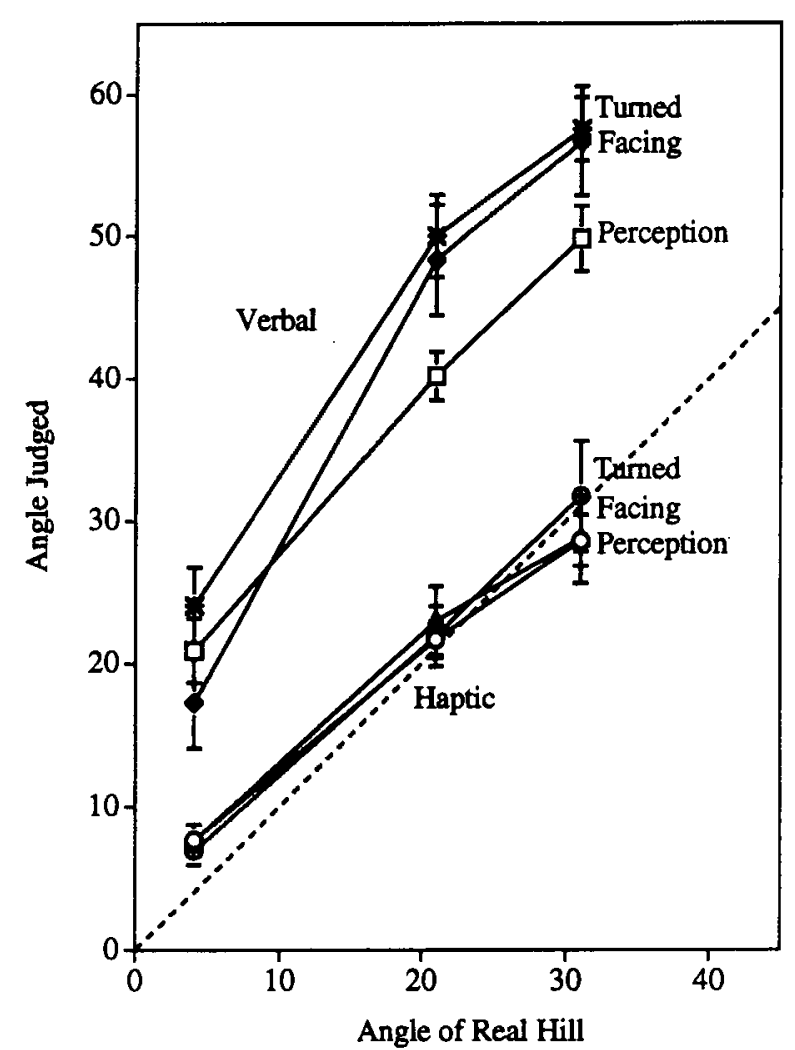

Figure 1. Mean verbal and haptic judgments $( \pm S E)$ in Experiment 1 for facing, turned, and perception conditions as a function of hill angle.

in a between-subjects design, with each subject judging only one hill. The normative data will be referred to as the perceptual condition. A four-way analysis of variance [(ANOVA) 3 (memory condition) $\times 3$ (angle) $\times 2$ (gender) $\times 2$ (verbal or haptic measure)] was performed on the combination of the present data set and the normative-data set, with hill measure as a within-subjects factor, and hill angle, memory condition, and gender as between-subjects factors.

As expected, the ANOVA showed a large main effect of hill measure. Slant was overestimated by verbal report but not by haptic adjustment. Figure 1 shows this consistent pattern in both the normative condition and the eyes-closed condition.

As predicted, there was a main effect for memory $[F(2,147)=3.38, p<.04]$. For the verbal measure, contrast comparisons revealed a significant difference between the perceived condition and the memorial conditions $[F(1,147)=9.43, p<.01]$ and revealed no difference between the two memorial conditions (facing and turned) $[F(1,147)=1.13, p<.30]$. The significant two-way interaction between hill measure and memory $[F(2,147)=$ $3.72, p<.03$ ] revealed that there was a difference between the perceived condition and the memory conditions on the verbal measure $[F(1,147)=9.43, p<.01]$, but there was no difference in the haptic measure $[F(1,147)=.07, p<.79]$. Memorial estimates were greater than perceived estimates only in the verbal measure.

The ANOVA also revealed an interaction between hill measure and gender $[F(1,147)=8.13, p<.01]$. Females gave higher verbal reports than males $[F(1,147)=6.89$, $p<.01]$, but there was no difference in haptic reports $[F(1,147)=0.71, p<.40]$. As in Proffitt et al. (1995), females overestimated in their verbal reports more than did males, but haptic adjustments remained the same for both males and females.

As in Proffitt et al. (1995), the data were plotted in both incremental coordinates and a log-log scale. As in the normative data, a power function provided an excellent fit for both the verbal and the haptic data as seen in the linear trend in a $\log -\log$ scale. The exponent of the power function was less than 1 , indicating a compression of sensitivity for slant in memory and in perception. The exponents for the perception and memory conditions were similar. In the perception condition: verbal, $y=11.72 x^{415}, R^{2}=.99$; haptic, $y=3.18 x^{636}, R^{2}=.99$. In the facing condition: verbal, $y=$ $7.65 x^{.593}, R^{2}=.99$; haptic, $y=3.14 x^{649}, R^{2}=.99$. In the turned condition: verbal, $y=13.30 x^{430}, R^{2}=.99$; haptic, $y=2.52 x^{726}, R^{2}=.99$.

\section{Discussion}

The mean judgments found in Experiment 1 show several important findings. First, verbal overestimations of slant were greater when slant was remembered than when it was seen. Second, there was no change in the haptic adjustments in memory. Third, there was no difference in verbal or haptic responses between the two conditions involving memory (facing the hill and turned around).

The results of Experiment 1 support several conclusions. First, immediate memory for slant replicates the data for perception of hills in which verbal judgments are greatly overestimated and haptic adjustments are much more accurate. In addition, we found a gender difference similar to that found by Proffitt et al. (1995). On the verbal measure, females overestimated more than did males; however, on the haptic measure, both genders performed the same. These findings suggest that judgments using visual memory are comparable to those made in visual perception.

For the memory effect, we found that verbal estimations of slant increased in memory. Second, motoric responses made from memory did not differ from those given when perceiving the hills. These findings suggest that, with a short delay between perception and response, memory for action and explicit memory remain separate. It appears that two separate memories inform action and awareness when given a short delay. These results are consistent with Proffitt et al.'s (1995) study, in which behavioral potential manipulations affected verbal but not haptic responses. In the short term, a manipulation may change one processing stream without affecting the other.

We found that there was no difference in the results between the facing and turned conditions. Although the turned 


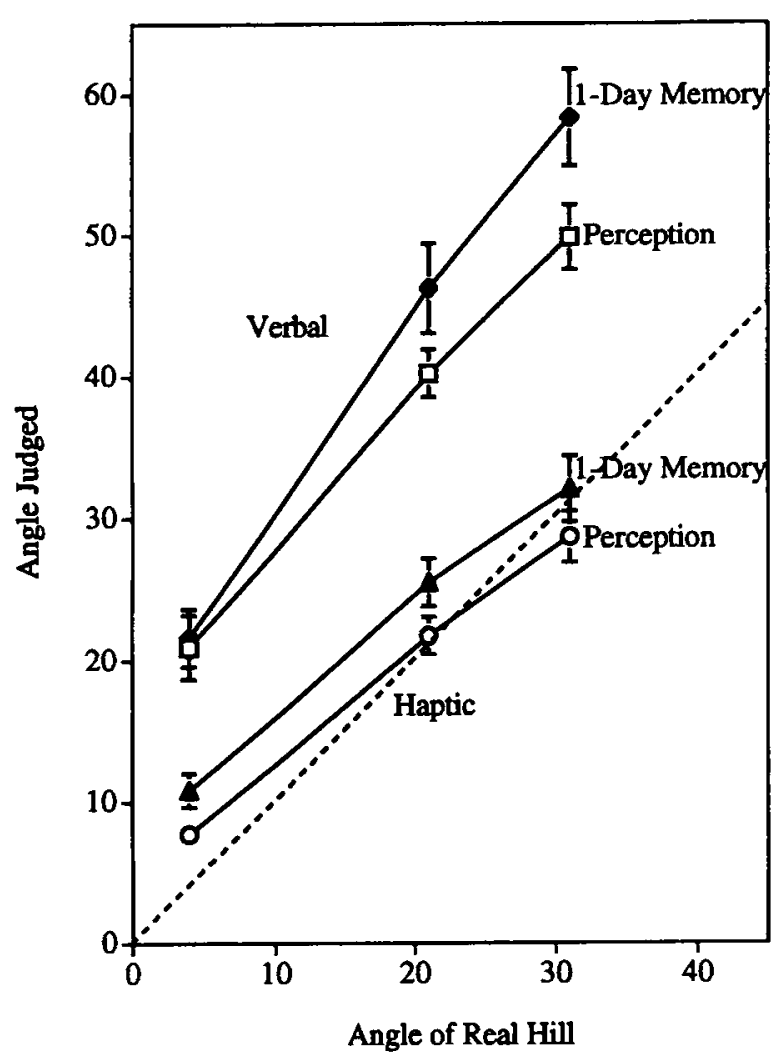

Figure 2. Mean verbal and haptic judgments $( \pm S E)$ in Experiment 2 for 1-day-delay and perception conditions as a function of hill angle.

condition involved a change in orientation, the motor response remained accurate even though the verbal response increased. We might expect from studies involving rotations and frame of reference that it would be more difficult to perform an egocentric action after a change in orientation (Reiser, 1989). However, in both memory conditions, the subjects remained in close proximity to the hill. This context may have allowed the subjects to preserve an accurate visual guidance memory that informed the action.

Memory for action and conscious awareness appear to be dissociated with a short delay; therefore, in Experiment 2 , we investigated memory for slant with a longer delay to see whether motor responses would increase along with verbal responses.

\section{EXPERIMENT 2 Judgments Made Away From the Hill After a Longer Delay}

In Experiment 1, verbal reports increased relative to the normative data, but haptic responses remained unchanged. These results suggest that, although explicit awareness of an incline increases in memory, an accurate visual guidance memory is preserved. The subjects still acted accurately, although their verbal responses were exaggerated, as we had expected. Experiment 1 required immediate mem- ory, where responses were made within $30 \mathrm{sec}$ of viewing the hill. In Experiment 2, the time delay between viewing and responding was changed. In Experiment 2, subjects viewed the hills one day and returned to a new location the next day to give their responses. Given previous research on the short-lived nature of visual guidance, it was predicted that both verbal and haptic judgments would be influenced by this delay.

\section{Method}

Subjects. Twenty students ( 9 males, 11 females) at the University of Virginia participated. They were volunteers who were paid for their participation. All subjects were naive to the purposes of this experiment and had not participated in any prior slant experiments. None had any apparent locomotor problems.

Stimuli and Apparatus. The same hills and tilt board were used as in Experiment 1 .

Design. Each subject viewed all three of the hills. All subjects reported their hill judgments in one of six counterbalanced orders. The order of verbal and haptic judgments was counterbalanced between subjects.

Procedure. On the first day, the subjects were told that they were taking part in a study on memory for different types of environments. They were told that they would be seeing three different locations and that they should try to remember these locations so that they would be able to describe the area to someone else and identify the specific place. They were told that they would be coming back to the laboratory the following day to answer questions about what they remembered in each environment. This premise was used so that, as in Experiment 1, the subjects would not explicitly evaluate the incline on the first day. The purpose was to see the hills, but then to recall the images and make judgments 1 day later. The subjects were taken to the three locations around the grounds of the University of Virginia.

On the second day, the subjects came into a quiet room to give their hill judgments. They sat down and were told that they would be asked questions about the places they had seen the day before. They were reminded of one of the locations and told to imagine what it looked like from their point of view, as if they were standing there. They were asked several questions about objects in the environment, as in Experiment 1. They were then asked to give the verbal and haptic judgments. For the haptic judgment, the tilt board was again placed at their side, and the measure was adjusted in the same way as it had been outside. The same procedure was used for all three hills.

\section{Results}

As in Experiment 1, a four-way ANOVA [ 2 (memory) $\times$ 3 (angle) $\times 2$ (gender) $\times 2$ (hill measure) $]$ was performed on the combination of this delayed-memory data set and the normative-data set, with hill measure as a within-subjects factor, and hill angle, memory condition, and gender as between-subjects factors. Although the memory data included hill angle as a within-subjects factor, and the normative data as a between-subjects factor, this analysis treated hill angle as a between-subjects factor to simplify the complex experimental design. This solution placed a stricter criterion on finding a significant difference between the two memory groups by reducing power.

The ANOVA revealed the consistent effect of hill measure $[F(1,138)=389.58, p<.0001]$, indicating that verbal judgments were much higher than haptic judgments. Most importantly, there was a main effect for memory condition $[F(1,138)=11.63, p<.0009]$, and there was no memory 
$x$ hill measure interaction. These results indicate that both verbal and haptic measures increased with the 1-day memory delay, relative to the perceptual condition. Figure 2 shows that, as verbal judgments increased, haptic judgments also increased.

Again, the plot in $\log -\log$ coordinates showed an excellent fit of the power function with similar exponents less than 1 in both the perception and the 1-day memory conditions. Perception: verbal, $\mathrm{y}=11.72 x^{.415}, R^{2}=.99$; haptic, $y=3.18 x^{636}, R^{2}=.99$. Memory: verbal, $y=11.09 x^{477}$, $R^{2}=.99$; haptic, $y=5.19 x^{526}, R^{2}=.99$.

\section{Discussion}

As predicted, in the 1-day delay condition, the subjects gave higher verbal and haptic estimates of the slant of hills. It appears that a length of time of 1 day is enough for the action system to require the input of the explicit memory system. When the explicit system has modified the representation of the incline, the action system follows and changes accordingly. In this case, when verbal judgments increased, haptic judgments also increased. These results support previous findings (Goodale, Jakobson, \& Keillor, 1994; Rossetti, in press) that actions performed without a visual stimulus may be driven by a conscious representation of the stimulus.

Although the haptic adjustments increased in Experiment 2 along with the verbal reports, they did not become the same as the verbal judgments. This finding is expected on the basis of Proffitt et al.'s (1995) internal consistency measure. Internal consistency results indicated that the subjects set the tilt board to $10^{\circ}$ both when looking at a $10^{\circ}$ hill that they perceived to be $30^{\circ}$ and when they were verbally instructed to set the board to $30^{\circ}$. Furthermore, with an instruction to set the board to $35^{\circ}$, they set it to $12^{\circ}$. In memory, the subjects remembered a $10^{\circ}$ hill to be $35^{\circ}$ instead of $30^{\circ}$. As the results of Experiment 2 show, this explicit memory informed a verbal response of $35^{\circ}$ and a motoric response of about $12^{\circ}$. Internal consistency would predict that, because explicit memory is informing both responses, they should increase together, but by different amounts. Experiment 3 replicated Experiment 2 with an even longer time frame.

\section{EXPERIMENT 3 General Memory Judgments for Hills}

The results of Experiment 2, unlike those of Experiment 1 , show a memory effect for both the verbal and the haptic responses. As the verbal reports increased from memory, the haptic responses also increased. A 1-day delay led to the necessary communication between the two systems. Experiment 3 investigated the influence of general memory for slants, on the basis of long-term experience with the hills. Since a delay of 1 day produced internally consistent verbal and haptic responses, it was predicted that general memory for these hills would produce the same effects. Subjects who were very familiar with the inclines that we used were asked to judge the hills from memory by imagining them within our laboratory.

\section{Method}

Subjects. Forty students ( 20 males, 20 females) at the University of Virginia participated in Experiment 3 to fulfill a course requirement. All subjects were naive to the purposes of this experiment and had not participated in any prior slant experiments. They were all full-time students who were very familiar with the grounds of the University of Virginia. None had any apparent locomotor problems.

Stimuli and Apparatus. The same hills and tilt board were used as in Experiments 1 and 2.

Design. Each subject imagined all three of the hills. All subjects reported their hill judgments in one of six counterbalanced orders. The order of verbal and haptic response was counterbalanced between subjects.

Procedure. The subjects were seated separately in a quiet room with the experimenter. They were told that the experimenter would describe several places around the grounds of the university. They were to imagine themselves at these places, and then, when they "arrived" at the specified place, they would be asked several questions about the place. Among more general questions, the subjects were asked to judge the angle of the incline with respect to the horizontal, giving a verbal and haptic measure. These judgments were recorded, and no feedback was given.

\section{Results}

A four-way ANOVA [ 2 (memory) $\times 3$ (angle) $\times 2$ (gender) $\times 2$ (hill measure) $]$ was performed on the combination of this general-memory data set and the normative-data set, with hill measure as a within-subjects factor, and hill angle, memory condition, and gender as between-subjects factors. As in Experiment 2, hill angle was treated as a between-subjects factor to compare with the normative data.

The ANOVA revealed the predicted effect of hill measure $[F(1,198)=548.82, p<.0001]$. As in Experiments 1 and 2 , verbal estimations were much higher than haptic judgments. Following our predictions, as in Experiment 2, there was a large main effect of memory $[F(1,198)=25.72$, $p<.0001]$. Furthermore, the absence of a memory $\times$ hill measure interaction indicates that the memory effect occurred for both verbal and haptic judgments as predicted. Figure 3 shows that, when verbal responses increased, haptic responses also increased, suggesting communication between the systems. The results also show a main effect for gender $[F(1,198)=12.97, p<.0004]$ and an interaction between hill measure and gender $[F(1,198)=27.53, p<$ $.0001]$. Females gave higher reports than did males only on the verbal measure $[F(1,198)=23.16, p<.0001]$, with no difference in haptic responses $[F(1,198)=0, p<.95]$.

The $\log -\log$ transformation showed again that the power function provided a good fit for the data. The exponents revealed somewhat smaller values for the memory condition, relative to the normative data. Perception: verbal, $y=11.72 x^{.415}, R^{2}=.99$; haptic, $y=3.18 x^{.636}, R^{2}=.99$. Memory: verbal, $y=18.10 x^{314}, R^{2}=.95$; haptic, $y=$ $8.79 x^{.375}, R^{2}=.97$.

A subsequent ANOVA performed on the verbal reports of Experiments 1, 2, and 3 revealed no difference between these responses. Although the verbal reports were consistently overestimated more than reports in the perception condition, there was not an increase in the overestimation with longer delays. 


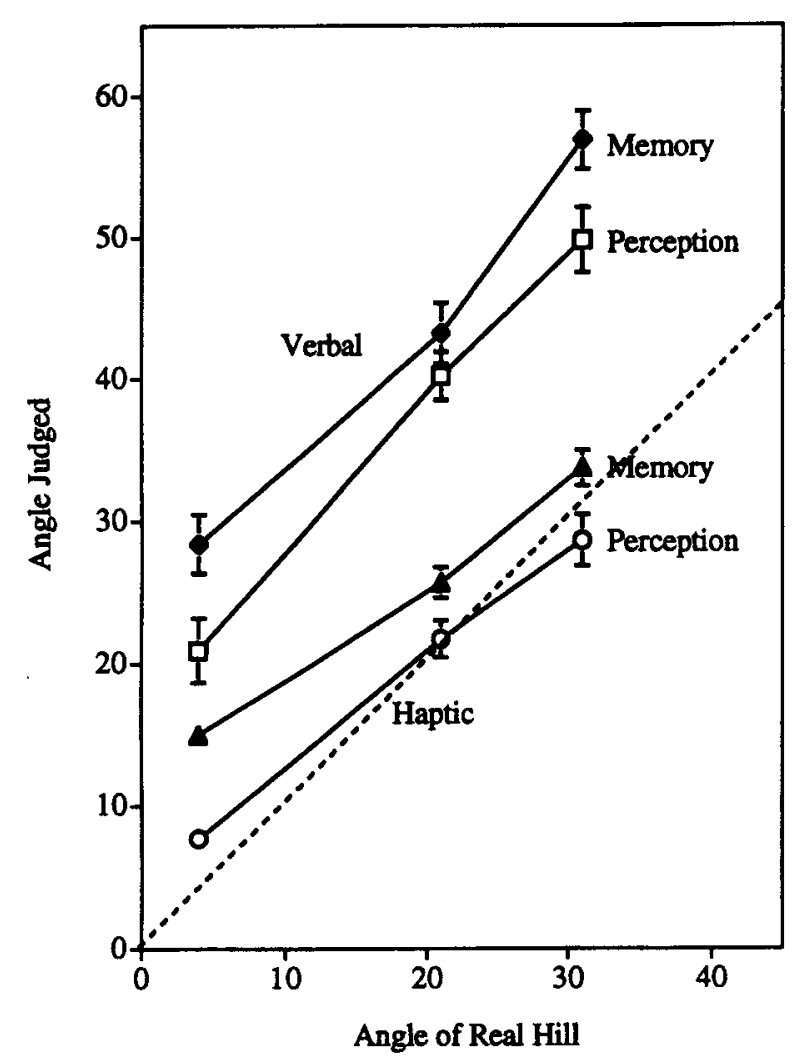

Figure 3. Mean verbal and haptic judgments $( \pm S E)$ in Experiment 3 for general memory and perception conditions as a function of hill angle.

\section{Discussion}

The results of Experiment 3 show again that, with a longer delay, the action system must be informed by a stored visual representation. The subjects were asked to remember an image of a hill they had seen often and then give verbal and haptic responses. As in Experiments 1 and 2, verbal judgments of slant increased in memory. Furthermore, haptic adjustments increased systematically with verbal reports. Again, as in Experiment 2, the increase in haptic response was proportional in terms of the internal consistency measure described earlier. These findings provide more evidence for the communication between explicit awareness and action.

\section{EXPERIMENT 4 \\ Judgments Made After a Short Delay Away From the Hill}

The results of Experiments 1-3 suggest that time is a deciding factor in influencing a change in motoric responses. Experiment 1 showed that, with a short delay, verbal reports of the incline of the hill increased, but haptic adjustments remained the same. Experiments 2 and 3, using a longer delay, showed an increase in both verbal and haptic responses. However, in Experiment 1, the subjects also remained in the same location while giving slant judg- ments from memory, whereas, in Experiments 2 and 3, the subjects changed locations. It is possible that the subjects were able to preserve a visual guidance memory only within the context of the relevant hill. Experiment 4 investigated whether changing the context in which one responded would lead to an increase in motoric responses along with verbal reports, even within the short time delay. Subjects completed the hill-judgment task either after walking to another location within $2 \mathrm{~min}$ or after standing in the same place with eyes closed for $2 \mathrm{~min}$.

\section{Method}

Subjects. Seventy students ( 27 males, 43 females) at the University of Virginia participated: 36 in the move condition, and 34 in the delay condition. They were volunteers who were given credit for their participation. All subjects were naive to the purposes of this experiment and had not participated in any prior slant experiments. None had any apparent locomotor problems.

Stimuli and Apparatus. The same hills and tilt board were used as in Experiments 1-3.

Design. Each subject viewed only one of the hills. In the move condition, there were 12 subjects for each of the hills. In the delay condition, there were 11 subjects for the $4^{\circ}$ hill, 12 for the $21^{\circ}$ hill, and 11 for the $31^{\circ}$ hill.

Procedure. The subjects viewed the hills binocularly from the front while standing at the base of the hills. They were instructed to look straight ahead and to try to remember the scene. They were told to be aware of the objects and landmarks in the environment and the layout of the land. The subjects were not explicitly told that they were to remember the slant of the hill, in order to prevent them from attaching an estimate to the incline while they were still looking at it.

After they had looked at the hill for about $10 \mathrm{sec}$, the subjects performed in one of two conditions. In the move condition, they walked with the experimenter to a new location, where the context of the hill and its immediate surroundings could no longer be seen. They were then asked to imagine the hill that they had seen and give verbal and haptic judgments in the manner of the previous experiments. The process of walking to a new location took no longer than $2 \mathrm{~min}$. In the delay condition, the subjects were instructed to close their eyes and answer several questions about what they had just seen for a period of $2 \mathrm{~min}$. Following these questions, the subjects were asked to imagine the hill and give haptic and then verbal estimates. The order of haptic and verbal responses was not counterbalanced for two reasons: First, there were no effects of order found in the previous experiments. Second, we wanted to ensure that giving a prior verbal report of an angle would not influence the haptic response.

\section{Results}

A four-way ANOVA [3 (memory condition: perception, move, or delay) $\times 3$ (angle) $\times 2$ (gender) $\times 2$ (hill measure)] was performed on the combination of this present data set and the normative-data set, with hill measure as a within-subjects factor, and hill angle, memory condition, and gender as between-subjects factors.

As in Experiments 1-3, the ANOVA showed a large main effect of hill measure $[F(1,142)=309.41, p<.001]$, in which verbal reports were greatly overestimated and motoric responses were much more accurate. Figure 4 shows this consistent pattern in both the perception condition and the move/delay conditions.

As predicted, there was a main effect for memory $[F(2,142)=7.78, p<.001]$ and a memory $\times$ hill measure interaction $[F(2,142)=3.19, p<.05]$. For the verbal mea- 


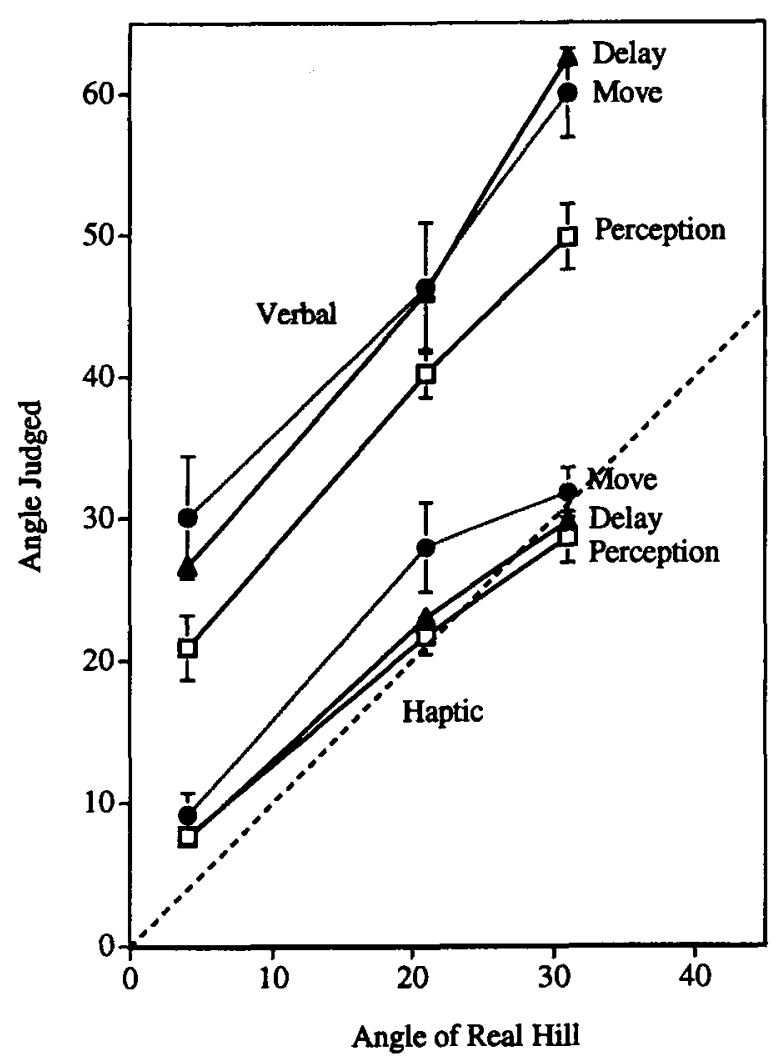

Figure 4. Mean verbal and haptic judgments $( \pm S E)$ in Experiment $\mathbf{4}$ for move, delay, and perception conditions as a function of hill angle.

sure, planned comparisons revealed a significant difference between the perception condition and the move condition $[F(1,142)=12.48, p<.001]$ and revealed no difference between the move and delay conditions $[F(1,142)=0.71$, $p<.40]$. These results indicate greater verbal overestimations in the two memory conditions than in the perception condition, as in Experiments 1-3. The effect of context, however, is seen in the results of the planned comparisons conducted for the haptic measure. The results showed that estimations in the move condition were greater than those in the perception condition $[F(1,142)=6.74, p<.01]$ and also greater than those in the delay condition $[F(1,142)=$ $4.13, p<.04]$. Figure 4 shows that verbal reports increased in both the move condition and the delay condition, but haptic responses increased proportionately only in the move condition. Leaving the context of the hill was necessary for an increase in haptic responses.

The ANOVA also revealed a main effect of gender $[F(1,142)=7.78, p<.001]$ and an interaction between hill measure and gender $[F(1,142)=8.23, p<.01]$. As in Experiments 1 and 3 , females gave larger verbal overestimations than did males $[F(1,142)=14.12, p<.001]$ but there was no difference in haptic adjustments between the two genders $[F(1,142)=2.13, p<.15]$.

\section{Discussion}

The results of Experiment 4 both replicate Experiment 1 and expand our understanding of the communication between the conscious awareness and visually guided action systems. In the delay condition, comparable to the short delay in Experiment 1, verbal overestimations increased relative to those in the perception condition, but haptic responses did not change. The move condition showed a proportional increase in both verbal and haptic responses, as in Experiments 2 and 3 with longer time delays. These results suggest that the short-term visual guidance memory system is tied to the spatial location in which it is formed. When the subjects were present at the hill in Experiment 4, as in Experiment 1, they showed an increase in their verbal report with no change in their motoric response. However, when the subjects were taken away from the hill, within the same short time delay, they relied on the explicit memory that informed both verbal and motoric responses. Experiment 4 shows that, in addition to time, a preservation of place is very important for accurate visually guided action.

\section{EXPERIMENT 4A Motor Interference}

Experiment 4 indicated that, with a short 2-min delay, motoric responses increased when the subject was taken to a new location but stayed the same when the subject remained in the presence of the hill. One possibility still open for question is whether it was simply the motoric act of walking that led to the disruption of an accurate visual guidance memory. It is possible that the increase in haptic response resulted from motor interference rather than a change of location. Experiment $4 \mathrm{~A}$ was designed to test this possibility by asking subjects for verbal and haptic responses for one hill after walking in place for $2 \mathrm{~min}$. It was assumed that the findings on one hill could be generalized to the other two.

\section{Method}

Subjects. Eleven University of Virginia students ( 5 males, 6 females) participated in the experiment. They were volunteers who were asked to participate as they walked by the hill. All subjects were naive to the purposes of this experiment and had not participated in any prior slant experiments. None had any apparent locomotor problems.

Stimuli and Apparatus. Only the $21^{\circ}$ hill was used. The same tilt board was used as in the previous experiments.

Design. Each subject viewed the one hill and gave a verbal and a haptic response. The order of response was not counterbalanced for reasons discussed in Experiment 4.

Procedure. The subjects viewed the hill under the same conditions as those in Experiment 4. After looking at the hill, they were instructed to turn around $180^{\circ}$ and face in the opposite direction. They were then instructed to march in place for $2 \mathrm{~min}$. After marching, the subjects were asked to remain facing in the same direction and to place their hand on the tilt board. They were asked to close their eyes and give the haptic response, as in the other experiments. Following their haptic response, they were instructed to remove their hand from the board and give a verbal estimate of the incline of the hill 


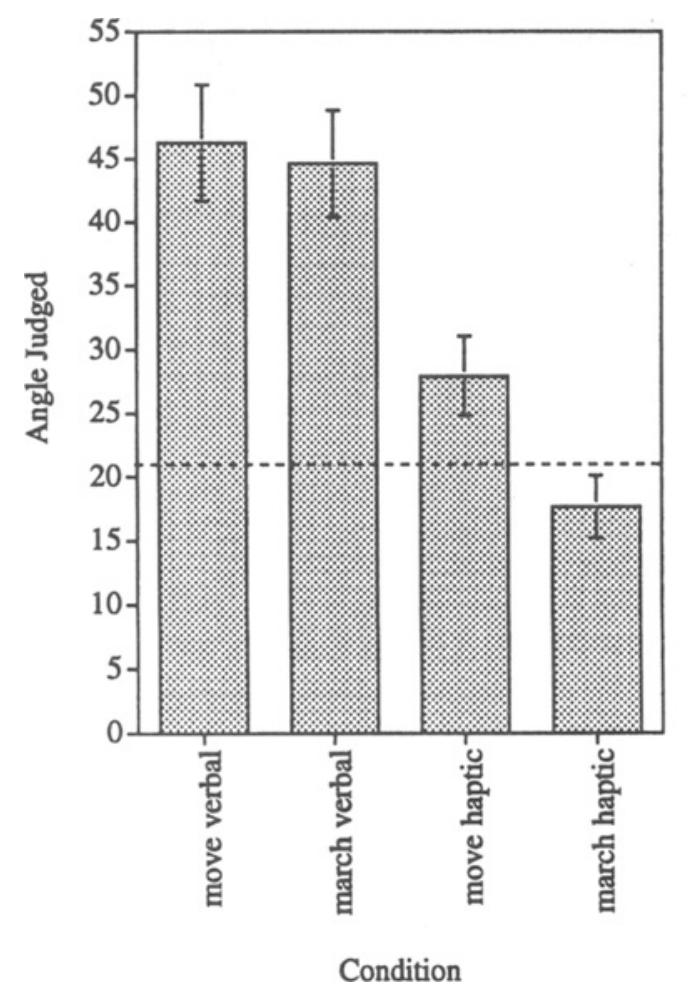

Figure 5. Mean verbal and haptic judgments $( \pm S E)$ in Experiment $4 \mathrm{~A}$ for the march and move conditions for the $21^{\circ}$ hill.

\section{Results}

Because only one hill was used in this experiment, independent $t$ tests were calculated between the move condition in Experiment 4 and the march condition of Experiment 4A for both verbal and haptic responses. These conditions both involved the same 2-min delay and a motor task during the delay. The only difference was that in the move condition, the subjects walked to another location, and, in the march condition, the subjects walked in place. The results indicated that, for the verbal response, there was no difference between the move and march conditions $[t(21)=0.26, p<.80]$. However, haptic responses were greater in the move condition than in the march condition $[t(21)=2.55, p<.02$; see Figure 5]. Taken together, verbal responses were overestimated in both the move condition and the march condition, but haptic adjustments increased only in the move condition. The subjects overestimated in their motoric responses after walking to a new location as discussed in Experiment 4, but they continued to be much more accurate after simply walking in place.

\section{Discussion}

The results of Experiment $4 \mathrm{~A}$ indicate that the increase in motoric response found when the subjects walked to a new location was not simply due to motor interference. In Experiment 4A, the motoric task of walking in place was used to create a similar movement without changing the subject's location. This condition allowed us to separate the effects of locomotion from a change in the environment. We found that, after the subjects walked in place for $2 \mathrm{~min}$, the haptic adjustment did not increase as it had when the subjects walked to a new location away from the hill. Thus, these results stress the importance of the context in which the response is made for accurate egocentric visually guided actions from memory.

\section{GENERAL DISCUSSION}

Our findings involving memory for geographical slant parallel the findings for perception of slant, in which verbal judgments are grossly overestimated and haptic adjustments are much more accurate. Most importantly, our results indicate the existence of two memory systems for hills that operate differently depending on the length of delay and response location. All of the experiments show that verbal memorial judgments of hills increased beyond the overestimation found when perceiving these hills. Furthermore, haptic responses from memory differed depending on the delay between perception and response and the place in which the response was made. With a short delay at the hill, haptic responses remained the same as responses made while perceiving the hills. With a long delay, haptic adjustments increased systematically with the verbal increase in overestimation. The importance of context for memorial visual guidance was shown in Experiment 4. Given a short time delay as in Experiment 1, the subjects showed an increase in motoric response only when moved to a new location away from the hill. These results suggest both separation between memories for visually guided action and explicit awareness (with a short delay in the context of the hill) and communication between explicit awareness and motor responses (with a long delay as well as responses after a short delay made in a new location). We suggest that the same explicit memory is informing the verbal slant judgments in all experiments, but different memory systems inform motor behaviors for short and long delays. Figure 6 shows that, with a short delay, the motoric response is guided by a visual guidance memory, whereas the verbal response is guided by a separate explicit memory. With a long delay or a short delay away from the hill, both motoric and verbal responses are guided by the same explicit memory.

The findings for memory for slant are consistent with the findings of Proffitt et al. (1995) that actions can be directly or indirectly influenced. Their study showed a measure of internal consistency suggesting an internal mapping between the awareness of slant and visually guided action. A person looking at a $10^{\circ}$ hill will report that it is $30^{\circ}$ but will motorically respond on the tilt board at $10^{\circ}$. The same person, when verbally instructed to set the tilt board to $30^{\circ}$, will set it to $10^{\circ}$. Thus, the motor adjustment of $10^{\circ}$ corresponds to an explicit awareness of $30^{\circ}$, whether both are informed by direct perception of the hill or explicit verbal instructions. Following this reasoning, in the internal con- 


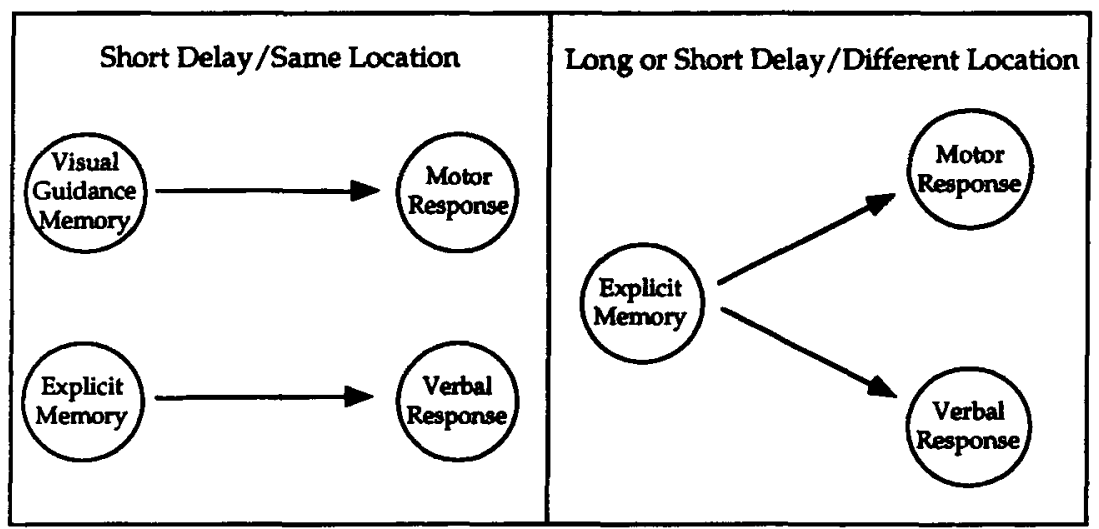

Figure 6. Models of memory for slant with short and long delays. With a short delay in the presence of the hill, memories are separate for awareness and action. Motor responses are guided by a visual guidance memory and verbal responses are guided by explicit memory (left panel). Given long or short delays and responses away from the hill, both motor and verbal responses are informed by explicit memory (right panel).

sistency measure, a verbal instruction to set the tilt board to $30^{\circ}$ or $35^{\circ}$ will lead a person to set it to about $10^{\circ}$ or $12^{\circ}$, respectively. In memory, a person explicitly remembers a $10^{\circ}$ hill to look $35^{\circ}$ instead of $30^{\circ}$. If the explicit memory is informing both verbal and haptic responses, then we would expect a verbal report of $35^{\circ}$ and a motoric response of about $12^{\circ}$. This is consistent with the results found in Experiments 2 and 3 with a long delay and the results of Experiment 4 with a short delay in a new location (see Figure 7).

\section{Increased Overestimations of \\ Phenomenal Awareness in Memory}

In all of our experiments, we found that verbal judgments of slant given from memory increased, relative to the judgments given when viewing the hills. In both perception and memory, judgments of geographical slant exhibit overestimation with response compression. As proposed in Proffitt et al. (1995), this property of conscious awareness of slant increases one's ability to distinguish between small differences in small slants. Since most of our walking is limited to small slants, it is more important for people to be sensitive to changes in small slants than to larger ones. A change from $8^{\circ}$ to $10^{\circ}$ should be much more noticeable than a change from $50^{\circ}$ to $52^{\circ}$. The apparent steepness of the hill informs one's decision of how to ascend the hill or possibly not to ascend it at all.

In memory, we see a similar response compression with a difference in the magnitude of overestimation. The fact that people increase memorial judgments of hills more

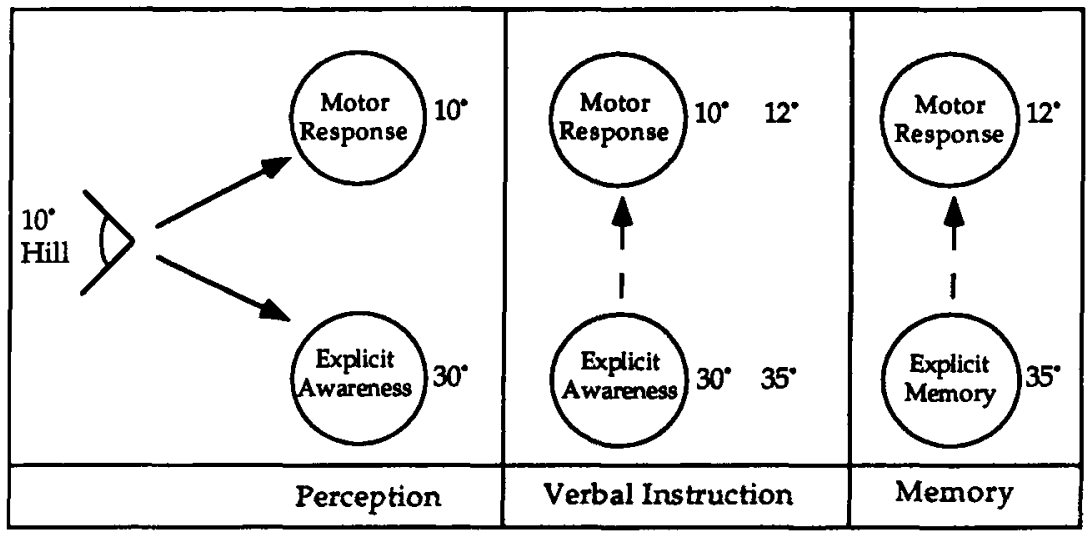

Figure 7. Model of internal consistency in perception and its relationship to memory. Proffitt et al. (1995) showed that a $10^{\circ}$ hill is judged verbally to be $30^{\circ}$. When a person is verbally instructed to set a tilt board to $30^{\circ}$ or $35^{\circ}$, he/she sets it to $10^{\circ}$ or $12^{\circ}$, respectively. In memory, a $10^{\circ}$ hill is remembered to be $35^{\circ}$ instead of $30^{\circ}$. It follows that the motor response, since informed by explicit memory, should rise to $12^{\circ}$ instead of $10^{\circ}$. 
than perceived judgments is an interesting phenomenon. The difference between the magnitudes of overestimation points to the different requirements and functions that perception and memory serve.

Other overestimations in memory have been found in magnitude estimates (Moyer, Bradley, Sorenson, Whiting, \& Mansfield, 1977). In a study of perceived and remembered size of objects, Moyer et al. found that estimates of length, area, and volume of objects were greater in memory, compared with estimates made while viewing objects. These overestimations, however, increased more for smaller magnitudes than for large ones, indicating a compression in memory. Similar results have been found in other memory psychophysics literature (Hubbard, 1994), although the results are somewhat variable.

Despite the lack of a definite explanation of why people increase their verbal reports of geographical slant from memory, our results are strong and extremely consistent. Even with a very short delay, the subjects gave higher verbal reports from memory, relative to the reports given while viewing the hills.

\section{Working Memory With \\ a Short delay at Hill Location}

With a short delay, motoric responses to hills did not increase, whereas verbal reports did increase. The change in one response and not the other suggests separate memories for phenomenal awareness and action. This proposal is supported by both neuroanatomical and behavioral research. Recent evidence suggests that the primate prefrontal cortex is segregated into different domains for memory (Wilson, O Scalaidhe, \& Goldman-Rakic, 1993). Wilson et al. found that dorsal prefrontal regions that are connected to dorsal stream visual areas (parietal cortex) show delay activity related to spatial information. Neurons in this area hold representations of spatial stimuli on line for brief periods when stimuli are no longer present. Ventral areas of the prefrontal cortex communicating to ventral visual areas encode cues for object characteristics. Wilson et al. proposed that information about object identity and location in memory may be processed separately from each other, corresponding to the distinction in the visual system. In addition, Ungerleider (1995) stated that the activity in prefrontal cortex during a delay is greater than in the inferior temporal cortex, and it does not suffer interference from the processing of other visual input. These results suggest that prefrontal cells maintain activity and then inform visual areas after a delay. Recent human fMRI data reported in Ungerleider also support this claim. In a working memory study, signals in the ventral occipitotemporal cortex depended on visual stimuli, but signals in the frontal lobe were sustained during the delay.

Looking at behavioral studies, Steenhuis and Goodale (1988) conducted a study that investigated short-term memory for spatial location. They asked subjects to walk to a target with their eyes closed, immediately after viewing the target or with 2-, 4-, or 30-sec delays. They concluded that short-term memory of target locations decays slowly over periods of time. There was no difference in performance for 2- or 4-sec delays, relative to performance with immediate walking. Even though subjects were less accurate after a 30-sec delay, the delay did not affect constant errors. Thus, they found more variability with time, but no systematic errors, suggesting that a memory for spatial location lasts over a short period of time of about $30 \mathrm{sec}$. Further studies of goal-directed locomotion have replicated the findings of Steenhuis and Goodale (Elliot, Jones, \& Gray, 1990), where 2-sec delays had no effect on performance, and more variable error resulted with a 30 -sec delay. Elliot et al. concluded that subjects remember general environmental layout, rather than a movement plan, and this representation deteriorates slowly over time.

\section{Explicit Memory With \\ a Delay and Change of Location}

After a delay of at least 1 day, motoric responses to hills increased along with verbal responses. In addition, the same results were found with a change of response location after a short delay. These findings suggest that a visual guidance memory lasts only a short amount of time and requires the context in which the stimulus was perceived. These results posit that, without the visual guidance memory informing the motor response, the action also must rely on the explicit memory that is informing the verbal response. Admittedly, our experiments of long delays also involve a change in location. Although we have not empirically shown that long delays without place changes result in a loss of visual guidance memory, we believe this must be so. Our findings support past research with normal subjects and neuropsychological patients, in which a delay led the action system to falter on its own. With a delay, normal subjects will change their visually guided actions to conform to conscious awareness (Goodale, Jakobson, \& Keillor, 1994; Rossetti \& Regnier, 1995). For example, saccades made to a remembered location will conform to the perceived location rather than the actual moved distance of the target (Wong \& Mack, 1981). Bridgeman et al. (1997) showed a similar effect using pointing and the Roelof's effect. After a delay, subjects' motor responses were influenced by the bias of the direction of the frame, as was found in the cognitive measure.

Recent work with actions made toward visual illusions has shown similar delay effects. The Müller-Lyer illusion, where a line is judged to be longer or shorter depending on the directions of arrows on the end, was used to test normal subjects (Gentilucci, Chieffi, Daprati, Saetti, \& Toni, 1996). Subjects were asked to point to one end of the line 0,2 , or $5 \mathrm{sec}$ after viewing the illusion. The results showed that subjects' pointing was slightly influenced by the illusion. When the line appeared longer, their pointing overestimated the endpoint; when the line appeared smaller, their pointing underestimated the endpoint. Furthermore, the illusion effect on pointing position increased with longer 
delays. By 5 sec, subjects clearly underestimated or overestimated the endpoint depending on the direction of the illusion. Gentilucci et al. proposed that, in no-vision conditions, the efficiency of egocentric cues is reduced, and environmental or contextual information interacts to guide movement.

Although there is a clear parallel between other research findings involving delayed actions to visual stimuli and the findings of our present experiments, there is a difference between the time scale involved. In our memory-forslant results, the motor response remained accurate with as long as a 2-min delay. In most of the research previously described, motor responses were influenced by conscious awareness within seconds of delay. However, we do find integration of explicit awareness and motor response with at least 1-day delay between seeing the hill and making responses and with a shorter time frame when response location is changed. One reason for this difference in time may be the stimulus used and the response made toward the stimulus. In the other studies, responses were pointing or saccade movements to small objects, which take a matter of seconds to complete. With geographical slant, the motor response is performed toward a large hill in terms of how one would walk up the hill. It may be that a memory for the environment, which then guides the potentially longer act of locomotion in the environment, may last longer than a memory to guide momentary action toward a small object. This proposal is supported by the results of Steenhuis and Goodale (1988), in which subjects in a memorial locomotion task were able to act relatively accurately with a 30 -sec delay.

\section{CONCLUSION}

Memory for geographical slant preserves the same relationship between phenomenal awareness and visually guided action as perception of geographical slant. Conscious verbal reports are greatly overestimated and motor responses are much more accurate. In memory, verbal reports show even more overestimation than did reports made while viewing hills, after both short and long delays. Motoric responses, however, differ depending on the delay and the place of response. With a short delay, motor responses at the hill do not change even though verbal responses increase. With a long delay, motor responses increase along with verbal responses. When taken away from the hill, a short delay also affects motoric estimates. These results taken together suggest both separation and communication in memory for visual guidance and explicit awareness. Motoric responses given after a short delay at the hill location are driven by a preserved visual guidance memory that operates in egocentric coordinates tied to the context of the hill. However, when a longer delay or a change of location is introduced, explicit memory informs both verbal and motoric responses. Therefore, in the short term, separate memories inform motor and verbal responses in the context of the stimulus. In the long term, without the short-lived visual guidance memory, explicit memory informs both motoric responses and explicit awareness. Our findings indicate a role for both time and place in the relative dissociation and interdependence between the two visual systems.

\section{REFERENCES}

Aglioti, S., DeSouza, J. F. X., \& Goodale, M. A. (1995). Sizecontrast illusions deceive the eye but not the hand. Current Biology, 5, 679-685.

Bhalla, M., \& ProffitT, D. R. (1997). Visual-motor recalibration in geographical slant perception. Manuscript submitted for publication.

Bridgeman, B., KirCh, M., \& Sperling, A. (1981). Segregation of cognitive and motor aspects of visual function using induced motion. Perception \& Psychophysics, 29, 336-342.

Bridgeman, B., Lewis, S., Heit, G., \& Nagle, M. (1979). Relation between cognitive and motor-oriented systems of visual position perception. Journal of Experimental Psychology: Human Perception \& Performance, 5, 692-700.

Bridgeman, B., PeEry, S., \& ANANd, S. (1997). Interaction of cognitive and sensorimotor maps of visual space. Perception \& Psychophysics, 59, 456-469.

Elliot, D., Jones, R., \& Gray, S. (1990). Short-term memory for spatial location in goal-directed locomotion. Bulletin of the Psychonomic Society, 28, 158-160.

Gentilucci, M., Chieffi, S., Daprati, E., Saetti, M. C., \& Toni, I. (1996). Visual illusion and action. Neuropsychologia, 34, 369-376.

GiBson, J. J. (1979). The ecological approach to visual perception. Boston: Houghton Mifflin.

Gibson, J. J., \& Cornsweet, J. (1952). The perceived slant of visual surfaces-optical and geographical. Journal of Experimental Psychology, 44, 11-15.

Goodale, M. A., Jakobson, L. S., \& Keillor, J. M. (1994). Differences in the visual control of pantomimed and natural grasping movements. Neuropsychologia, 32, 1159-1178.

Goodale, M. A., Jakobson, L. S., Milner, A. D., Perrett, D. I., BenSON, P. J., \& HiEtANEN, J. K. (1994). The nature and limits of orientation and pattern processing supporting visuomotor control in a visual form agnosic. Journal of Cognitive Neuroscience, 6, 46-56.

GoOdale, M. A., \& Milner, A. D. (1992). Separate visual pathways for perception and action. Trends in Neurosciences, 15, 20-25.

Goodale, M. A., Milner, A. D., Jakobson, L. S., \& Carey, D. P. (1991). A neurological dissociation between perceiving objects and grasping them. Nature, 349, 154-156.

Hubbard, T. L. (1994). Memory psychophysics. Psychological Research, 56, 237-250.

Jakobson, L. S., ArChibald, Y. M., Carey, D. P., \& Goodale, M. A. (1991). A kinematic analysis of reaching and grasping movements in a patient recovering from optic ataxia. Neuropsychologia, 29, 803-809.

KammanN, R. (1967). The overestimation of vertical distance and slope and its role in the moon illusion. Perception \& Psychophysics, 2, 585589.

Kinsella-Shaw, J. M., Shaw, B., \& Turvey, M. T. (1992). Perceiving "walk-on-able" slopes. Ecological Psychology, 4, 223-239.

Milner, A. D., \& GoOdale, M. A. (1995). The visual brain in action. Oxford: Oxford University Press.

Moyer, R., Bradley, S., Sorenson, M., Whiting, J., \& MansFIELD, D. (1977). Psychophysical functions for perceived and remembered size. Science, 200, 330-332.

Pelisson, D., Prablanc, C., Goodale, M. A., \& Jeannerod, M. (1986). Visual control of reaching movements without vision of the limb. Experimental Brain Research, 62, 303-311

Proffitt, D. R., Bhalla, M., Gossweiler, R., \& Midgett, J. (1995). Perceiving geographical slant. Psychonomic Bulletin \& Review, 2, 409-428. 
REISER, J. J. (1989). Access to knowledge of spatial structure at novel points of observation. Journal of Experimental Psychology: Learning, Memory \& Cognition, 15, 1157-1165.

Ross, H. E. (1974). Behavior and perception in strange environments. London: George Allen \& Unwin.

RosSETTI, Y. (in press). Implicit perception in action: Short-lived motor representations of space. In P. G. Grossenbacher (Ed.), Finding consciousness in the brain: A neurocognitive approach (Advances in Consciousness Research). Philadelphia: J. Benjamins.

Rossetti, Y., \& Regnier, C. (1995). Representations in action: Pointing to a target with various representations. In B. G. Bardy, R. J. Boutsma, \& Y. Guiard (Eds.), Studies in perception and action III (pp. 233-239). Hillsdale, NJ: Erlbaum.

Steenhuis, R. E., \& Goodale, M. A. (1988). The effects of time and distance on accuracy of target-directed locomotion: Does an accurate short-term memory for spatial location exist? Journal of Motor Behavior, 20, 399-415.
UNGERLEIDER, L. G. (1995). Functional brain imaging studies of cortical mechanisms for memory. Science, 270, 769-775.

UNGERLEIDER, L. G., \& MishKIN, M. (1982). Two cortical visual systems. In D. J. Ingle, M. A. Goodale, \& R. J. W. Mansfield (Eds.), Analysis of visual behavior (pp. 549-586). Cambridge, MA: MIT Press.

Wilson, F. A. W., O Scalaidhe, S. P., \& Goldman-Rakic, P. S. (1993). Dissociation of object and spatial processing domains in primate prefrontal cortex. Science, 260, 1955-1958.

WONG, E., \& MACK, A. (1981). Saccadic programming and perceived location. Acta Psychologica, 48, 123-131.

Wraga, M., Creem, S. H., \& Proffit, D. R. (in press). Spatial frames of reference in perceiving and acting. In M. McBeath (Ed.), Navigational principles in humans, animals, and machines. Thousand Oaks, CA: Sage.

(Manuscript received June 10, 1997; revision accepted for publication August 21, 1997.) 